\title{
Ocular perfusion pressure and intraocular pressure in pregnant women with severe preeclampsia
}

\author{
Haldun Arpacı ${ }^{1}$, Yaran Koban², Abdullah Tok ${ }^{3}$, Abdullah Beyoğlu \\ 'Department of Obstetrics and Gynecology, Kafkas University, School of Medicine, Kars, Turkey \\ ${ }^{2}$ Department of Ophthalmology, Kafkas University, School of Medicine, Kars, Turkey \\ ${ }^{3}$ Department of Obstetrics and Gynecology, Kahramanmaras Sutcuimam University School of Medicine, Kahramanmaras, Turkey \\ ${ }^{4}$ Department of Ophthalmology, Kahramanmaras Sutcuimam University School of Medicine, Kahramanmaras, Turkey
}

\begin{abstract}
Objectives: The aim of this study was to investigate whether severe preeclampsia (SPE) affects intraocular pressure (IOP) and ocular perfusion pressure (OPP).

Material and methods: This prospective and comparative study included 64 pregnant females, allocated into 2 groups as Group 1 (31 pregnant women with SPE) and Group 2 (33 normotensive pregnant women). IOP, systolic and diastolic blood pressure, OPP of all the subjects were measured after 20 weeks of gestation and prior to labor and medical therapy.

Results: The mean IOP values in Group 1 were not significantly different from those of Group 2 ( $p=0.528$ ). The mean OPP values in Group 1 were significantly higher than those of Group $2(p<0.001)$. There was no significant correlation between IOP and OPP levels. No significant differences were determined between the groups in respect of age, gestational age body mass index (BMI) $(p<0.269, p<0.219$ and $p<0.556$ respectively). The mean systolic and diastolic blood pressure values were statistically significantly higher in Group 1 than in Group $2(p<0.001$ and $p<0.001)$.

Conclusions: Although high IOP was expected in SPE patients, it was found to be constant. This could have been due to an increase in aqueous humor outflow because of changes occurring in angiogenic and anti-angiogenic factors in SPE.

Key words: severe preeclampsia, intraocular pressure, ocular perfusion pressure
\end{abstract}

Ginekologia Polska 2018; 89, 9: 513-517

\section{INTRODUCTION}

Preeclampsia (PE) is defined as a pregnancy-specific hypertensive disease, developing in the 2 nd or 3rd trimester as a complication of $2 \%-7 \%$ of pregnancies [1]. It refers to the new onset of hypertension and proteinuria or end-organ dysfunction after 20 weeks of gestation in previously normotensive women [2] and is a leading cause of maternal and perinatal morbidity and mortality [3]. SPE is one of the worst sequlae of hypertensive disorders in pregnancy. SPE with estimated incidence of $0.6 \%-1.2 \%$ [4], is an uncommon hypertensive disorder in pregnancy that is characterized by excessive maternal systemic inflammation and endothelial dysfunction [5]. This results in temporary ischemic injury of many body organ systems.
The visual system may be affected to different degrees in PE. Ocular symptoms in patients with PE are caused by various underlying pathological changes in the retina, cerebral cortex, and optic nerve [6]. Recent studies have shown that women with a history of PE remain at higher risk for ocular complications than healthy pregnant women during the intrapartum and postpartum period [7,8]. Although most of the ocular changes in pregnancy are physiological, in cases with PE these may be pathological.

Although ocular changes in women with PE have been investigated in many studies, there remains a need for further studies to determine ocular disorders that emerge in PE [9]. In these previous studies, SPE and mild preeclamptic cases have been evaluated in the same pool and to the best 
of our knowledge there has been no study that has evaluated IOP and OPP in isolated SPE patients. There have been found to be conflicting results in the data obtained from studies conducted on PE patients for other purposes. The aim of this study was to investigate the possible effect of SPE on IOP and OPP during pregnancy and thereby contribute to literature.

\section{MATERIAL AND METHODS}

This multi-center prospective case-control study was conducted by the Department of Gynecology and Obstetrics Departments and Ophthalmology Departments of Kafkas University Medical Faculty Hospital (KUMFH) and Kahramanmaras Sutcu Imam University Medical Faculty Hospital (KSUMFH). The study protocol adhered to the tenets of the Declaration of Helsinki and approval was granted by the KUMFH Institutional Eethics Committee (date: 25 October 2017). The study included all consecutive patients after the 20th week of gestation who were treated in the KUMFH and KSUMFH for SPE between November 2017 and April 2018. All subjects provided informed consent for participatione in the study.

\section{Patient selection}

The study included 31 pregnant women complicated by SPE (Group 1) and 33 healthy pregnant women (Group 2) matched for age, gestational age and BMI. BMI was calculated from height and weight measurements. The 31 SPE patients and 33 healthy pregnant women, aged 18-40 years, were recruited from the antenatal clinics of the above-mentioned hospitals. None of the participants were in active labor or had received any volume expansion or magnesium sulphate therapy before the ophthalmic examination.

SPE diagnosis was made according to the following criteria [2]: The Task Force on Hypertension in Pregnancy defined SPE as severe hypertension [after 20 weeks of gestation with a previously normal blood pressure, systolic blood pressure of $\geq 160 \mathrm{~mm} \mathrm{Hg}$ or diastolic blood pressure of $\geq 110 \mathrm{~mm} \mathrm{Hg}$ on two occasions at least 4 hours apart while the patient is on bed rest (unless antihypertensive therapy is initiated before this time)] and proteinuria [ $\geq 300 \mathrm{mg}$ per 24-hour urine collection (or this amount extrapolated from a timed collection) or protein/creatinine ratio $\geq 0.3$ or dipstick reading of $1+]$ in the absence of proteinuria with new-onset hypertension [after 20 weeks of gestation with a previously normal blood pressure, systolic blood pressure of $\geq 140 \mathrm{~mm} \mathrm{Hg}$ or diastolic blood pressure of $\geq 90 \mathrm{~mm} \mathrm{Hg}$ on two occasions at least 4 hours apart] with the new onset of any of the following features: thrombocytopenia [platelet count $<100,000 / \mu \mathrm{L}$ ], progressive renal insufficiency [serum creatinine concentration $>1.1 \mathrm{mg} / \mathrm{dL}$ or a doubling of the serum creatinine concentration in the absence of other renal disease], pulmonary edema, new-onset of cerebral or visual disturbances and impaired liver function as indicated by abnormally elevated blood concentrations of liver enzymes (to twice normal concentration), severe persistent right upper quadrant or epigastric pain unresponsive to medication and not accounted for by alternative diagnoses, or both.

Patients were excluded if they had twin pregnancy, gestational hypertension, chronic hypertension, mild preeclampsia, renal disease, diabetes mellitus, gestational diabetes mellitus, overt cardiovascular disease or if they smoked. Women with a history of glaucoma, glaucomatous optic disc changes, papillary hemorrhage, glaucomatous visual field defects, IOP readings $>22 \mathrm{~mm} \mathrm{Hg}$, any findings associated with elevated IOP (shallow anterior chamber, pseudoexfoliation syndrome), high spherical and ciliary refractive errors (myopia, hypermetropia, astigmatism), or a history of previous intraocular surgery or laser therapy were also excluded.

\section{IOP and OPP measurements}

To avoid the effects of seasonal variations on IOP, this study was conducted in the winter season [10]. As previous studies have demonstrated a circadian rhythm of aqueous humor flow the IOP and OPP measurements in the current study were taken in the morning in both the patients and control groups, thereby avoiding potential bias. After resting for at least 15 minutes, brachial blood pressure was recorded using an automated oscillometric machine from the upper arm, with the patient in a seated position. IOP is dynamic and determined by several variables. The relationship between IOP and these variables can be modeled by the modified Goldmann equation: IOP $=\mathrm{EVP}+(\mathrm{Q}-\mathrm{U}) / \mathrm{c}$; where EVP is the episcleral venous pressure, $Q$ is the aqueous humor flow rate, $\mathrm{c}$ is the conventional outflow facility, and $U$ is the pressure-insensitive uveoscleral outflow rate [11]. IOP was measured at least 3 times using an I-Care rebound tonometer (ICare TA01; Tiolat, Helsinki, Finland). The mean value was used for analysis [12]. OPP was calculated based on mean arterial pressure (MAP) and IOP according to the following formula: mean OPP $=2 / 3$ (MAP-IOP). MAP was obtained from systolic blood pressure (SBP) and diastolic blood pressure (DBP) using the following formula: $M A P=D B P+1 / 3(S B P-D B P)$

The IOP and OPP of all subjects both in SPE and control groups were measured after the 20th week of gestation. The measurements of all patients with SPE were evaluated prior to active labor or volume expansion therapy or magnesium sulphate therapy. The IOP measurements of both eyes of each participant were taken with the same I-Care rebound tonometer. The measurements of the right eyes were included in this study. Full ophthalmologic evaluations, including best-correct visual acuity, slit-lamp and dilated 
fundus evaluation, were performed when the general state was stable.

\section{Statistical analysis}

The data of the right eye of each patient were analysed. All statistical analyses were performed using Statistical Package for the Social Sciences Software, version 24.0 (SPSS Inc., Chicago, IL. USA) program. The conformity of the data to normal distribution was confirmed using the Kolmogorov-Smirnov test. The Mann-Whitney $U$ test was used to compare variables between groups. Pearson's correlation was used to examine the relationships between IOP and OPP. The results were given as mean \pm standard deviation (SD) and $p<0.05$ was considered statistically significant.

\section{RESULTS}

The clinical and demographic characteristics of the 31 SPE women (Group 1) and 33 normotensive pregnant women (Group 2) are summarized in Table 1. There were no significant differences between the groups in respect of age $(30.23 \pm 2.88$ years vs $31.30 \pm 4.68$ years; $p=0.269)$, gestational age $(33.45 \pm 2.81$ weeks vs $32.27 \pm 4.61$ weeks; $p=0.219)$ and BMI $(31.52 \pm 2.40$ v.s $31.12 \pm 2.95 \mathrm{p}=0.556)$. Group 1 had a significantly higher systolic blood pressure $(159.84 \pm 6.38 \mathrm{~mm}$ $\mathrm{Hg}$ vs $114.24 \pm 5.01 \mathrm{~mm} \mathrm{Hg} ; \mathrm{p}<0.001)$, diastolic blood pressure $(98.55 \pm 6.73 \mathrm{~mm} \mathrm{Hg}$ vs $75.15 \pm 5.07 \mathrm{~mm} \mathrm{Hg} ; \mathrm{p}<0.001)$ and MAP $(118.50 \pm 6.45 \mathrm{~mm} \mathrm{Hg}$ vs $88.15 \pm 3.92 \mathrm{~mm} \mathrm{Hg}$; $\mathrm{p}<0.001$ ) than Group 2. The mean IOP values in Group 1 were not significantly different from those of Group $2(14.32 \pm 3.87$ vs $13.79 \pm 2.80 ; p=0.528)$. The mean OPP values in Group 1 were significantly higher than those of Group 2 (69.42 \pm 5.38 vs $48.34 \pm 6.42 ; p<0.001)$. There was no significant correlation between IOP and OPP levels. No significant correlation was determined between the measured parameters, and the IOP, and OPP values. Although reported in literature, no cases of vitreous hemorrhage, serous retinal detachment, Purstcher like retinopathy or cortical blindness were encountered in this study.

\section{DISCUSSION}

$\mathrm{PE}$ is a multifactorial disease, characterized by systemic endothelial dysfunction [5]. The effects of PE are different from the effects of hypertension alone, which is the most basic feature [13]. During PE, both endothelial derived growth factors and pro-inflammatory molecules may lead to vasculopathic ophthalmic conditions. As changes in angiogenic and anti-angiogenic factors in SPE [14] could cause an increase in aqueous humor outflow and because of the balance between these two conditions, it is thought that IOP could remain constant. If the changes in these factors are responsible for the IOP remaining constant, the measurement of IOP could be a marker for preeclampsia that is simple to apply. Unlike previous studies where mild and SPE cases have been studied together, only women with SPE were evaluated in the current study. Both the SPE patients and the control group were in the third trimester of pregnancy. In the comparison of the SPE patients with the normotensive women while OPP values were found to be significantly higher in the SPE patients no significant difference was seen in the IOP values.

Due to the major physiological changes in pregnancy, changes are seen in IOP, corneal thickness, and some visual functions such as accommodation and visual field [15]. In a recent meta-analysis, IOP values of healthy pregnant women in the third trimester have been shown to be lower than those of non-pregnant women [16]. The physiological

\begin{tabular}{|c|c|c|c|}
\hline Variables & $\begin{array}{l}\text { Pregnant women with severe } \\
\text { preeclampsia } \\
\text { (Group 1) } \\
\text { n: } 31\end{array}$ & $\begin{array}{l}\text { Normotensive pregnant } \\
\text { women } \\
\text { (Group 2) } \\
\text { n: } \mathbf{3 3}\end{array}$ & $\mathbf{p}^{\mathbf{a}}$ \\
\hline Age [years] & $30.23 \pm 2.88$ & $31.30 \pm 4.68$ & 0.269 \\
\hline $\mathrm{BMI}\left[\mathrm{kg} / \mathrm{m}^{2}\right]$ & $31.52 \pm 2.40$ & $31.12 \pm 2.95$ & 0.556 \\
\hline Gestational age [week] & $33.45 \pm 2.81$ & $32.27 \pm 4.61$ & 0.219 \\
\hline Systolic Blood pressure [mmHg] & $159.84 \pm 6.38$ & $114.24 \pm 5.01$ & $<0.001^{*}$ \\
\hline Diastolic Blood pressure [mm Hg] & $98.55 \pm 6.73$ & $75.15 \pm 5.07$ & $<0.001^{*}$ \\
\hline MAP & $118.50 \pm 6.45$ & $88.15 \pm 3.92$ & $<0.001^{*}$ \\
\hline IOP & $14.32 \pm 3.87$ & $13.79 \pm 2.80$ & 0.528 \\
\hline OPP & $69.42 \pm 5.38$ & $48.34 \pm 6.42$ & $<0.001^{*}$ \\
\hline
\end{tabular}

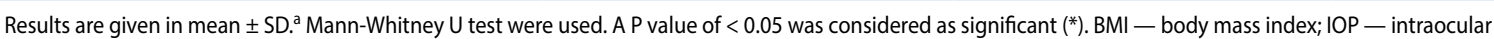
pressure; MAP — mean arterial pressure; OPP — ocular perfusion pressure 
mechanisms responsible for the decrease of IOP during pregnancy are not clearly known [17]. Due to a rise in the levels of progesterone during pregnancy either directly or indirectly cause an increase in fluid outflow from the eye without altering the rate of fluid entry. $2-3 \mathrm{~mm} \mathrm{Hg}$ decrease in episcleral venous pressure during pregnancy may affect fluid outflow from the eye $[18,19]$.

Pregnancy is known to have a beneficial impact on many ocular defects including glaucoma $[10,20]$. Hence, physiological changes in pregnancy may normalize IOP levels in women with ocular hypertension [21]. In agreement with this, decreased IOP has been reported in both normotensive [20] and hypertensive pregnant women [10]. It has also been reported that pregnancy leads to a decline in IOP of up to $20 \%$ in healthy normotensive women [21]. Likewise, in pregnant subjects with ocular hypertension, pregnancy leads to a decline in IOP of up to $25 \%$ [21].

Although there is no specific research in literature related to the effect of SPE on IOP and OPP, conflicting results have been reported. Qureshi et al reported that the mean IOP values of hypertensive pregnant women in the third trimester were statistically significantly higher than those of patients who were not hypertensive. However, that study excluded patients with PE [10]. In a study by Giannia et al, the IOP values of preeclamptic women were reported to be increased in the peripartum period compared to normotensive pregnant women. Patients with SPE were not evaluated in that study, and unlike the our study, patients in the postpartum period were included [22]. Philips et al. found no difference in third trimester IOP values between hypertensive and non-hypertensive pregnant women. However, in that study there were no data whether pregnant patients had PE [23]. The IOP and OPP findings reported by Sayın et al of pregnant women with PE were similar to our results [24].

High systolic blood pressure causes high IOP [25-27]. In contrast, IOP levels did not rise and remained constant in pregnant women with SPE. Factors affecting the formation of IOP may be responsible for unchanged IOP levels. Aqueous humor flow rate is the most important contributor of aqueous humor formation [11]. It has been reported that aqueous humor flow could be affected with the effect of episcleral venous pressure associated with high pressure $[28,29]$. Normal IOP levels in SPE cases are suggesting the lack of resistance to aqueous humor outflow throughout the episcleral pathways.

Hormonal milieu, endothelial damage, hypoperfusion ischemia, hyperperperfusion edema and abnormal autoregulation may affect ocular changes in PE [20]. The most frequently seen ophthalmological abnormalities in preeclamptic women are retinal artery spasm [30] and orbital vascular hyperperfusion [31]. The prevalence of vascular spasm and resistance in preeclampsia patients is expected to cause an increase in upper extremity venous pressure. Hence, both episcleral venous pressure and IOP are expected to rise. In addition, an enhancement in the production of aqueous humor in preeclamptic patients is thought to be a result of both increased retinal blood flow and siliceous cell blood flow. However, as the IOP was determined to be constant in the preeclampsia patients in the current study, a significant enhancement in the aqueous humor flow or reduction in the production of aqueous humor may keep the IOP values constant.

\section{CONCLUSIONS}

In conclusion, constant IOP levels in SPE patients may be due to increased aqueous humor outflow. In the light of these findings, there is a need for further studies to reach firmer conclusions about the impact of SPE on aqueous humor flow.

\section{Conflict of interest}

The authors declare that there are no conflicts of interest.

\section{REFERENCES}

1. Mol B, Roberts C, Thangaratinam S, et al. Pre-eclampsia. The Lancet. 2016; 387(10022): 999-1011, doi: 10.1016/s0140-6736(15)00070-7.

2. Hypertension in Pregnancy. Obstetrics \& Gynecology. 2013; 122(5): 1122-1131, doi: 10.1097/01.aog.0000437382.03963.88.

3. Saving Mothers' Lives: Reviewing maternal deaths to make motherhood safer: 2006-2008. BJOG: An International Journal of Obstetrics \& Gynaecology. 2011; 118: 1-203, doi: 10.1111/j.1471-0528.2010.02847.x.

4. Sibai B. Evaluation and management of severe preeclampsia before 34 weeks' gestation. American Journal of Obstetrics and Gynecology. 2011; 205(3): 191-198, doi: 10.1016/j.ajog.2011.07.017.

5. C.Weel I, Romão-Veiga $M$, Matias $M$, et al. Increased expression of NLRP3 inflammasome in placentas from pregnant women with severe preeclampsia. Journal of Reproductive Immunology. 2017; 123: 40-47, doi: 10.1016/j.jri.2017.09.002

6. Prado R, Figueiredo $E$, Magalhães T. Retinal Detachment in Preeclampsia. Arquivos Brasileiros de Cardiologia. 2002; 79(2): 185-186, doi: 10.1590/s0066-782x2002001100011.

7. Murphy M, Casselman R, Smith G. Postpartum alterations in circulating endothelial progenitor cells in women with a history of pre-eclampsia. Pregnancy Hypertension: An International Journal of Women's Cardiovascular Health. 2013; 3(3): 178-185, doi: 10.1016/j.preghy.2013.03.004.

8. Kvehaugen AS, Dechend R, Ramstad HB, et al. Endothelial Function and Circulating Biomarkers Are Disturbed in Women and Children After Preeclampsia. Hypertension. 2011; 58(1): 63-69, doi: 10.1161/hypertensionaha.111.172387.

9. Lupton SJ, Chiu CL, Hodgson L, et al. Changes in Retinal Microvascular Caliber Precede the Clinical Onset of Preeclampsia. Hypertension. 2013; 62(5): 899-904, doi: 10.1161/hypertensionaha.113.01890.

10. Qureshi I,XiX, WuX.Intraocular pressure trends in pregnancy and in the third trimester hypertensive patients. Acta Obstetricia et Gynecologica Scandinavica. 1996; 75(9): 816-819, doi: 10.3109/00016349609054709.

11. Goel M. Aqueous Humor Dynamics: A Review !2010-03-03 !2010-06-17 !2010-09-02 ! The Open Ophthalmology Journal. 2010; 4(1): 52-59, doi: 10.2174/1874364101004010052.

12. Barbosa C, Stefanini F, Penha F, et al. Intraocular pressure and ocular perfusion during hemodialysis. Arquivos Brasileiros de Oftalmologia. 2011; 74(2): 106-109, doi: 10.1590/s0004-27492011000200007.

13. Garg A, Wapner R, Ananth C, et al. Choroidal and Retinal Thickening in Severe Preeclampsia. Investigative Opthalmology \& Visual Science. 2014; 55(9): 5723, doi: 10.1167/iovs.14-14143.

14. Levine R, Maynard S, Qian C, et al. Circulating Angiogenic Factors and the Risk of Preeclampsia. New England Journal of Medicine. 2004; 350(7): 672-683, doi: 10.1056/nejmoa031884. 
15. Sunness J. The pregnant woman's eye. Survey of Ophthalmology. 1988; 32(4): 219-238, doi: 10.1016/0039-6257(88)90172-5.

16. Changes in intraocular pressure and central corneal thickness during pregnancy: a systematic review and Meta-analysis. International Journal of Ophthalmology. 2017, doi: 10.18240/ijo.2017.10.15.

17. WEINREB R, LU A, KEY T. Maternal Ocular Adaptations during Pregnancy. Obstetrical \& Gynecological Survey. 1987; 42(8): 471-483, doi: 10.1097/00006254-198708000-00001.

18. Green K, Phillips C, Cheeks L, et al. Aqueous Humor Flow Rate and Intraocular Pressure during and after Pregnancy. Ophthalmic Research. 1988; 20(6): 353-357, doi: 10.1159/000266751.

19. Wilke K. Episcleral venous pressure and pregnancy. Acta Ophthalmologica. 2009; 53(S125): 40-41, doi: 10.1111/j.1755-3768.1975.tb01228.x.

20. Dinn R, Harris A, Marcus P. Ocular Changes in Pregnancy. Obstetrical \& Gynecological Survey. 2003; 58(2): 137-144, doi: 10.1097/01.ogx.0000047741.79433.52.

21. Qureshi IA. Intraocular pressure and pregnancy: a comparison between normal and ocular hypertensive subjects. Arch Med Res. 1997; 28(3): 397-400, indexed in Pubmed: 9291638.

22. Giannina G, Belfort M, Abadejos P, et al. Comparison of intraocular pressure between normotensive and preeclamptic women in the peripartum period. American Journal of Obstetrics and Gynecology. 1997; 176(5): 1052-1055, doi: 10.1016/s0002-9378(97)70401-0.

23. Phillips $\mathrm{Cl}$, Gore SM. Ocular hypotensive effect of late pregnancy with and without high blood pressure. British Journal of Ophthalmology. 1985; 69(2): 117-119, doi: 10.1136/bjo.69.2.117.
24. Sayin N, Kara N, Pirhan D, et al. Subfoveal Choroidal Thickness in Preeclampsia: Comparison with Normal Pregnant and Nonpregnant Women. Seminars in Ophthalmology. 2013; 29(1): 11-17, doi: 10.3109/08820538.2013.839813.

25. LESKEM, PODGORM. INTRAOCULAR PRESSURE, CARDIOVASCULAR RISK VARIABLES, AND VISUAL FIELD DEFECTS. American Journal of Epidemiology. 1983; 118(2): 280-287, doi: 10.1093/oxfordjournals.aje.a113634.

26. Bill A. The role of ciliary blood flow and ultrafiltration in aqueous humor formation. Experimental Eye Research. 1973; 16(4): 287-298, doi: 10.1016/0014-4835(73)90094-8.

27. Klein B. Intraocular pressure and systemic blood pressure: longitudinal perspective: the Beaver Dam Eye Study. British Journal of Ophthalmology. 2005; 89(3): 284-287, doi: 10.1136/bjo.2004.048710.

28. Bill A. Blood circulation and fluid dynamics in the eye. Physiological Reviews. 1975; 55(3): 383-417, doi: 10.1152/physrev.1975.55.3.383.

29. Physiology of the Eye: Clinical Application. Journal of the American Medical Association. 1960; 172(12): 1340, doi: 10.1001/jama.1960. 03020120118034.

30. HALLUM A. EYE CHANGES IN HYPERTENSIVE TOXEMIA OF PREGNANCY Journal of the American Medical Association. 1936; 106(19): 1649, doi: 10.1001/jama.1936.02770190025009.

31. OHNOY, KAWAI M, WAKAHARAY, et al. Ophthalmic Artery Velocimetry in Normotensive and Preeclamptic Women With or Without Photophobia. Obstetrics \& Gynecology. 1999; 94(3): 361-363, doi: 10.1097/00006250199909000-00008.z 